\title{
The ISPS/MIPE 2018 editorial for the joint conference on Information Storage and Processing Systems and Micromechatronics for Information and Precision Equipment, San Francisco, California, USA
}

\author{
Kenji Fukuzawa ${ }^{1} \cdot$ Shaomin Xiong ${ }^{2} \cdot$ Yung-Kan Chen $^{3}$
}

Published online: 29 November 2019

(C) Springer-Verlag GmbH Germany, part of Springer Nature 2019

It is our great pleasure to host the special issue for the joint International Conference on Information Storage and Processing Systems and Micromechatronics for Information and Precision Equipment (ISPS/MIPE 2018). This year conference was the 27 th annual conference for ISPS.

In American Society of Mechanical Engineers (ASME), the ISPS Division is largely devoted to hard disk drives, tape drives, optical drives, and other storage and information related devise to name a few. ISPS has its annual conference held in California USA (previously at Santa Clara and recently at San Francisco). In Japan Society of Mechanical Engineers (JSME), the Information, Intelligence and Precision Equipment (IIP) Division is the major division working on information storage systems, intelligent machines, precision machines, bio-medical equipment, etc. IIP has its annual

Kenji Fukuzawa

fukuzawa@nuem.nagoya-u.ac.jp

Shaomin Xiong

Shaomin.Xiong@wdc.com; xshaomin@berkeley.edu

Yung-Kan Chen

Yungkan.chen@wdc.com

1 Department of Micro/Nano Systems Engineering, Nagoya University, Nagoya, Japan

2 Western Digital Corporation Research, Milpitas, CA, USA

3 Western Digital Corporation, San Jose, CA, USA

conference held in Japan. ISPS and IIP agreed to hold the joint MIPE conference every 3 years alternating between Japan and US. Both ISPS and IIP merged their own annual conferences into MIPE so that participants from both conferences get a chance to meet together. Thus far, the joint MIPE conference was held at Yokohama, Japan in 2003, at Santa Clara, USA in 2006, at Tsukuba, Japan in 2009, at Santa Clara, USA in 2012, and at Kobe, Japan in 2015, respectively. This year the conference was held at San Francisco, USA.

The ISPS/MIPE 2018 conference was very successful. It had attracted more than 50 top quality presentations from all over the world, mainly the Asia pacific area, presented in multiple breakout sessions that covered the ISPS and IIP technical disciplines, mainly focused in the area of data storage devices and precision equipment. Many papers presented in the conference had affiliations from both academia and industry, driven by a very close and effective industry-university research collaborations.

As a tradition of ASME ISPS conference, this year we granted five ISPS Division graduate student fellowships, featured by a monetary award, and four ISPS Division graduate student scholarships, featured by a conference fee waiver. Students who got the award must demonstrate continuous contribution to the ISPS and show high level of research quality. The purpose of the award is to encourage participation of our next generation engineers and researchers who might change the industry in future.

Authors of recommended presentations in the conference were invited and encouraged to submit full papers with extended contribution to this special issue. All the submissions have gone through a rigorous peer-review process, like a regular paper. We sincerely thank all 
reviewers who helped to review the manuscripts in a thorough and timely manner.

We would like to acknowledge the help from conference staff, all track and session organizers and the ISPS executive members. We would like to have our special thanks to the ASME ISPS Division chair Prof. Paul C.-P. Chao and vice chair Dr. Andrey Ovcharenko, Conference chair Dr. Aravind Murthy and Prof. Gen Hashiguchi, for their great contributions and leadership in this conference. Last, but not least, a final word of gratitude goes to all presenters and participants of the conference.

Guest Editors:

Prof. Kenji Fukuzawa

Dr. Shaomin Xiong

Dr. Yung-Kan Chen

Publisher's Note Springer Nature remains neutral with regard to jurisdictional claims in published maps and institutional affiliations. 